\title{
Stationary echo canceling in velocity estimation by time-domain cross-correlation
}

\author{
Jensen, Jørgen Arendt
}

Published in:

I E E E Transactions on Medical Imaging

Link to article, DOI:

10.1109/42.241874

Publication date:

1993

Document Version

Publisher's PDF, also known as Version of record

Link back to DTU Orbit

Citation (APA):

Jensen, J. A. (1993). Stationary echo canceling in velocity estimation by time-domain cross-correlation. I E E E Transactions on Medical Imaging, 12(3), 471-477. https://doi.org/10.1109/42.241874

\section{General rights}

Copyright and moral rights for the publications made accessible in the public portal are retained by the authors and/or other copyright owners and it is a condition of accessing publications that users recognise and abide by the legal requirements associated with these rights.

- Users may download and print one copy of any publication from the public portal for the purpose of private study or research.

- You may not further distribute the material or use it for any profit-making activity or commercial gain

- You may freely distribute the URL identifying the publication in the public portal

If you believe that this document breaches copyright please contact us providing details, and we will remove access to the work immediately and investigate your claim. 


\title{
Stationary Echo Canceling in Velocity Estimation by Time-Domain Cross-Correlation
}

\author{
Jørgen Arendt Jensen
}

\begin{abstract}
The application of stationary echo canceling to ultrasonic estimation of blood velocities using time-domain crosscorrelation is investigated. Expressions are derived that show the influence from the echo canceler on the signals that enter the cross-correlation estimator. It is demonstrated that the filtration results in a velocity-dependent degradation of the signal-to-noise ratio. An analytic expression is given for the degradation for a realistic pulse. Due to the inherent nonlinear method of velocity estimation, it is most appropriate to state a probability of correct detection at low signal-to-noise ratios. This probability is influenced by signal-to-noise ratio, transducer bandwidth, center frequency, number of samples in the range gate, and number of A-lines employed in the estimation. Quantitative results calculated by a simple simulation program are given for the variation in probability from these parameters. An index reflecting the reliability of the estimate at hand must be calculated from the actual cross-correlation estimate, as the probability is influenced by velocity and signal-to-noise ratio. It is shown that such an index can be calculated by a simple formula and used in rejecting poor estimates or in displaying the reliability of the velocity estimated.
\end{abstract}

\section{INTRODUCTION}

$\mathbf{T}$ HE INVESTIGATION of the hemodynamics of the human body has been greatly eased by the introduction of pulsed ultrasound systems for measuring blood velocities. By these an image of the flow in the body can be displayed in realtime showing, e.g., the function of the heart valves, flow in the veins, and possible stenosis. The non-ionizing investigation is painless and relatively inexpensive compared to other imaging modalities.

Most of the current scanners are based on an estimation of a frequency shift of the emitted pulse spectrum [1]. This is essentially a phase measurement and gives rise to the depth-velocity limitation [2]. This can be circumvented by the time-domain cross-correlation approach [3]-[6]. Here the high-frequency sampled signal from successive received pulseecho lines (A-lines) are cross-correlated, and the velocity is found by locating the maximum peak in the cross-correlation function. The approach also has the advantage that broad band pulses can be used, so the scanner is optimized in the same way for both B-mode and velocity images.

One problem in this technique, however, is that the time domain estimator does not always pick out the correct peak in the estimate of the cross-correlation function. This is mainly due to noise in the acquired signals, which distorts

Manuscript received August 30, 1991; revised February 9, 1993.

The author is with the Electronics Institute, Technical University of Denmark, DK-2800 Lyngby, Denmark.

IEEE Log Number 9210286. the cross-correlation function estimate. The distortion depends on the signal-to-noise ratio, which can be quite low (0-20 dB) for clinical data. At large depths in tissue, and thus large attenuation, it is, therefore, most appropriate to state a probability of correct detection.

In this paper we will study the effect of including stationary echo canceling prior to the velocity estimation. The canceler removes echoes from stationary objects such as the tissue and boundaries surrounding the blood stream. The canceling is done by subtracting successive lines. The influence of doing so is studied in the next section. It is shown that the transfer function of the stationary echo canceling filter depends on velocity, and that this makes the signal-to-noise ratio velocitydependent. This will affect the probability of correct detection as shown in Section III. Graphs of the change in probability, when the signal-to-noise ratio, velocity, transducer bandwidth, number of lines, and segment length are varied, are shown.

As the velocity and signal-to-noise ratio are unknown $a$ priori, an evaluation of the estimate must be based on the actual data. An index directly related to the probability of correct detection can be calculated from the estimated correlation function as shown in Section IV. This index can be smoothed and then used for rejecting unreliable estimates and for displaying the reliability of the estimates shown on the scanner screen.

\section{Stationary Echo CANCELING}

The purpose of stationary echo canceling is to remove echoes from the boundaries of the veins and from the surrounding tissue, so only the flow signal enters the velocity estimator. This is a necessity as the amplitude of the stationary signal often is a factor of 10 to 100 larger than the flow signal. The removal is done by filtering the successively acquired Alines using the data values at the same time instance relative to the pulse emission from the transducer as shown in Fig. 1.

The A-line signals received consist of a flow signal $y_{f}$ and a stationary signal $y_{s}$ from the tissue:

$$
y_{i}(t)=y_{s_{i}}(t)+y_{f_{i}}(t)
$$

where $i$ denotes the A-line number. In [4] it was shown that a flow results in a time displacement between consecutively received signals of

$$
t_{s}=\frac{\Delta z}{c / 2}=f \frac{2 T_{p r f}|\vec{v}| \cos (\Theta)}{c}
$$

where $c$ is the ultrasound propagation velocity, $\Delta z$ is the distance away from the transducer traveled between pulse 


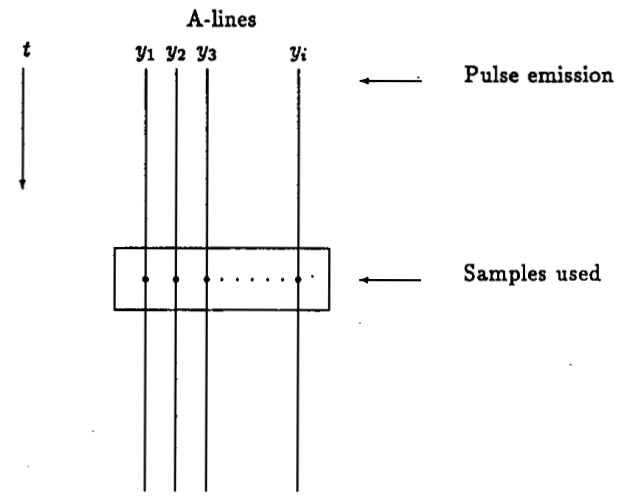

Fig. 1. Calculation of stationary echo canceling.

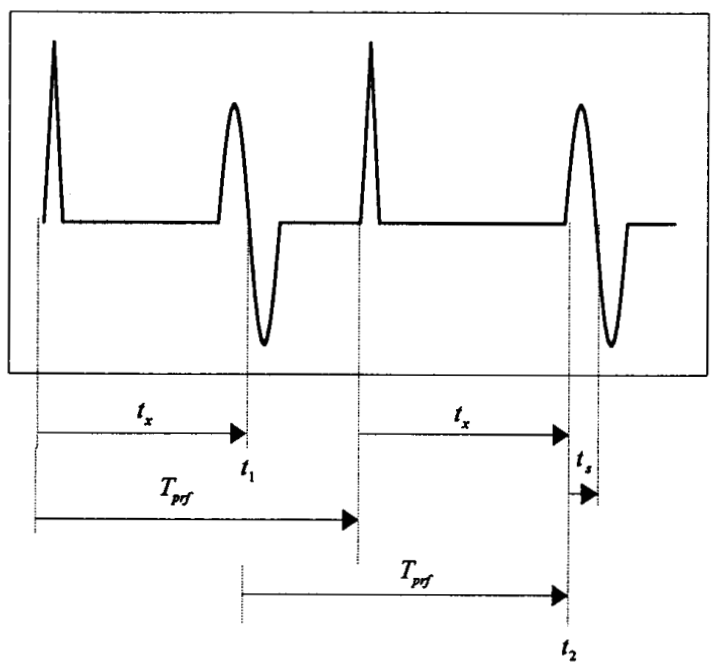

Fig. 2. Time intervals between signals for two pulse emissions.

emissions, $|\vec{v}| \cos \Theta$ is the velocity in direction away from the transducer, $\Theta$ is the angle between the flow vector and the ultrasound beam, and $T_{p r f}=1 / f_{p r f}$ is the time between emission of pulses.

The timing for two pulse emissions is shown in Fig. 2. The time from pulse emission to sampling is $t_{x}$ and pulses are emitted every $T_{p r f}$ seconds. The responses are received at $t_{1}$ and $t_{2}=t_{1}+T_{p r f}$. Due to the movement of scatterers, the response is shifted $t_{s}$ seconds, when the second pulse is emitted, so the received signals are related by

$$
y_{f_{2}}\left(t_{2}\right)=y_{f_{1}}\left(t_{2}-T_{p r f}-t_{s}\right)=y_{f_{1}}\left(t_{2}-t_{s}\right) \text {. }
$$

The last equality is valid because the same tissue is probed at periodic intervals. Using (3), eq. (1) can be written as

$$
\begin{aligned}
y_{i}(t)= & y_{s_{1}}\left(t-T_{p r f} \cdot(i-1)\right) \\
& +y_{f_{1}}\left(t-T_{p r f} \cdot(i-1)-t_{s} \cdot(i-1)\right) .
\end{aligned}
$$

As the measurement is repeated every $T_{p r f}$ seconds we have

$$
y_{s_{i}}(t)=y_{s_{1}}\left(t-T_{p r f} \cdot(i-1)\right)=y_{s_{1}}(t)
$$

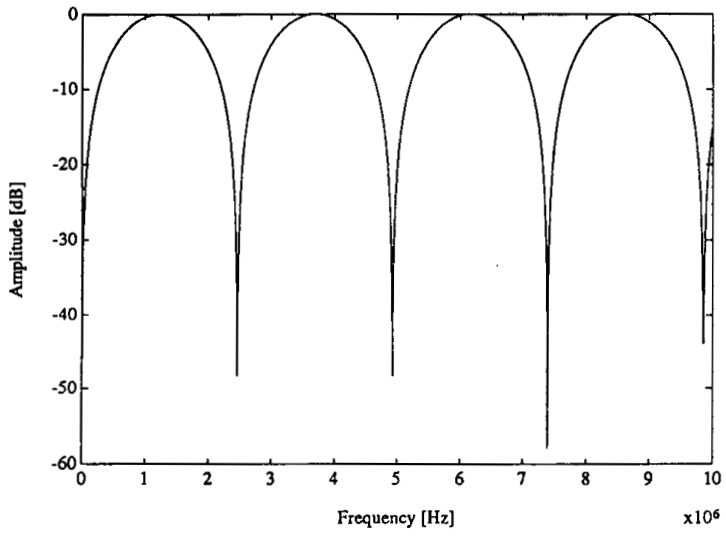

Fig. 3. Transfer function of stationary echo canceling filter at $v=1 \mathrm{~m} / \mathrm{s}$.

$$
\begin{aligned}
y_{f_{i}}(t) & =y_{f_{1}}\left(t-T_{p r f} \cdot(i-1)-t_{s} \cdot(i-1)\right) \\
& =y_{f_{1}}\left(t-t_{s} \cdot(i-1)\right) .
\end{aligned}
$$

The last equality is valid because the same scatterers are interrogated for each pulse. Further, a laminar flow is assumed; and noise, attenuation, and diffraction effects are neglected. A simple method for removing $y_{s}$ from $y$ is to subtract two consecutive lines as suggested in [4]. The technique is also used in the autocorrelation approach to velocity estimation [1], where it is called a delay line canceler in consistency with the radar literature [7]. Using the simple FIR filter results in

$$
\begin{aligned}
e_{s_{1}}(t) & =\frac{1}{2}\left(y_{s_{1}}(t)-y_{s_{2}}(t)\right)=\frac{1}{2}\left(y_{s_{1}}(t)-y_{s_{1}}(t)\right)=0 \\
e_{f_{1}}(t)=\frac{1}{2}\left(y_{f_{1}}(t)-y_{f_{2}}(t)\right) & =\frac{1}{2}\left(y_{f_{1}}(t)-y_{f_{1}}\left(t-t_{s}\right)\right) .
\end{aligned}
$$

Fourier transforming the last expression yields

$$
\begin{aligned}
H(f) & =\frac{E_{f}(f)}{Y(f)}=0.5\left(1-\exp \left(j 2 \pi f t_{s}\right)\right) \\
& =0.5\left(1-\exp \left(j 2 \pi f v \frac{2 T_{p r f}}{c}\right)\right) \\
& =0.5\left(1-\exp \left(j 2 \pi f / f_{s h}\right)\right) \\
f_{s h} & =\frac{c}{2 v} f_{p r f}
\end{aligned}
$$

where $H(f)$ is the Fourier transform of the filter, and $E_{f}$ and $Y$ are the transforms of $e_{f}$ and $y$. The transfer function of the filter depends on the velocity of the blood. $f_{s h}$ can be regarded as a variable sampling frequency depending on the blood velocity. The transfer function of the filter at a velocity of $1 \mathrm{~m} / \mathrm{s}$ is shown in Fig. 3. The pulse repetition frequency $f_{p r f}$ was $3.2 \mathrm{kHz}$ and the propagation velocity $1540 \mathrm{~m} / \mathrm{s}$. This makes $f_{s h}$ equal to $2.46 \mathrm{MHz}$, and the zeros in the transfer function at multiple of this sampling frequency are seen. The number of zeros introduced into the spectral range of the pulse depends on the velocity. This is shown in Fig. 4, where the position of zeros in the spectrum is tabulated as a function of velocity. The consequence of the filtration of the flow signal is a reduction in amplitude and a distortion of the pulse spectrum that depends on velocity. 


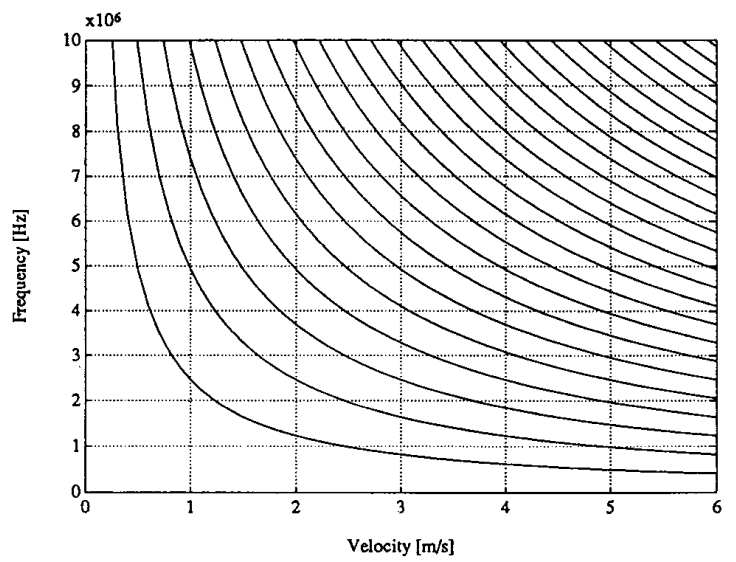

Fig. 4. Position of zeros as a function of blood velocity.

The reduction in signal-to-noise ratio can be quantified by introducing models for the signals involved. A useful model for the received signal is

$$
y(t)=\int_{-\infty}^{\infty} p\left(t-t^{\prime}\right) s\left(t^{\prime}\right) d t^{\prime}+n(t)=p(t) * s(t)+n(t)
$$

$p(t)$ is the pulse echo impulse response of the ultrasound system including the electromechanical impulse response of the transducer. The pulse can also contain the attenuation of the ultrasound by the intervening tissue. $s(t)$ is a white, zero mean scattering signal with a Gaussian amplitude distribution. This corresponds to the scattering signal from the blood. $n(t)$ is white, zero mean noise with a Gaussian amplitude distribution. The noise is assumed independent of $s(t)$ and of the noise in the other lines acquired. The covariance of the noise is the same from line to line.

The signal-to-noise ratio is defined as

$$
\mathrm{snr}=\sqrt{\frac{E\left[(p(t) * s(t))^{2}\right]}{E\left[n^{2}(t)\right]}}
$$

where $E$ is the expectation operator. The signal-to-noise ratio for the filtered signal is

$$
\begin{aligned}
\mathrm{snr} & =\sqrt{\frac{E\left[\left\{p(t) * s(t)-p(t) * s\left(t-t_{s}\right)\right\}^{2}\right]}{E\left[\left\{n_{1}(t)-n_{2}(t)\right\}^{2}\right]}} \\
& =\sqrt{\frac{E\left[\left\{p(t) *\left(s(t)-s\left(t-t_{s}\right)\right)\right\}^{2}\right]}{E\left[n_{1}^{2}(t)\right]+E\left[n_{2}^{2}(t)\right]}} \\
& =\frac{1}{\sqrt{2}} \sqrt{\frac{E\left[\left\{p(t) * h\left(t ; t_{s}\right) * s(t)\right\}^{2}\right]}{E\left[n^{2}(t)\right]}}
\end{aligned}
$$

where $h\left(t ; t_{s}\right)$ is the impulse response of the stationary echo canceling filter, whose response depends on the delay time $t_{s}$.

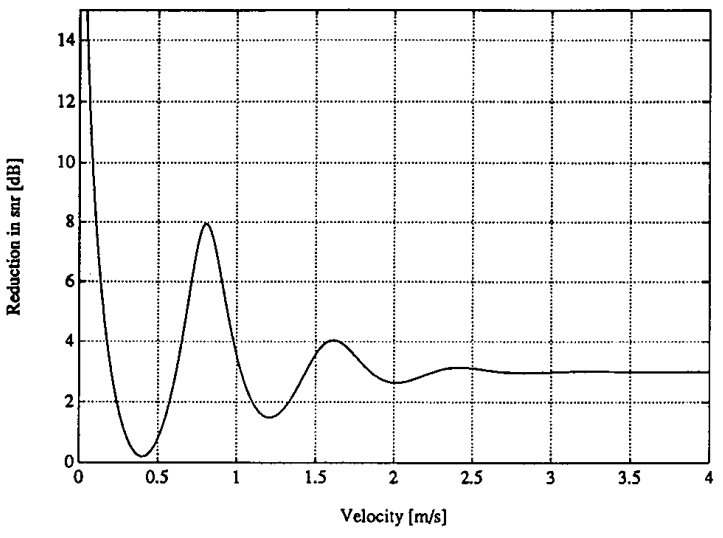

Fig. 5. Reduction of the signal-to-noise ratio due to the stationary echo canceling filter as a function of velocity.

From (10) it can be seen that the filtering results in a 3-dB loss in signal-to-noise ratio at high velocities, and that the loss will vary with velocity. The loss will depend on the shape of the pulse spectrum and the center frequency. An example of a pulse could be

$$
p(t)=\exp \left(-2\left(B_{r} f_{0} \pi\right)^{2} t^{2}\right) \cos \left(2 \pi f_{0} t\right)
$$

where $B_{r}$ is the relative bandwidth and $f_{0}$ the center frequency. The pulse is non-causal and non-minimum phase, but this is of no importance as the cross-correlation function calculated here is phase-blind.

Using (10) and (11) the reduction in signal-to-noise ratio is calculated in Appendix A to be (12) [at bottom of page].

The reduction in signal-to-noise ratio as a function of velocity is shown in Fig. 5, when using $f_{0}=3 \mathrm{MHz}, B_{r}=0.2$, and $f_{p r f}=3.2 \mathrm{kHz}$. A notable reduction is seen at low velocities, where the loss can be dramatic, due to the zero in the filter when $v=0 \mathrm{~m} / \mathrm{s}$. The reduction in signal-to-noise ratio can be minimized by selecting a large bandwidth, as the pulse then is short so little part of it overlaps from A-line to A-line. This is demonstrated in Fig. 6, where the reduction is shown as a function of bandwidth for $v=0.8 \mathrm{~m} / \mathrm{s}$.

Until now only the simple subtraction filter has been investigated. More advanced filters can be employed. In general the transfer function is

$$
H_{i}(f)=\sum_{k=1}^{N_{a v e}} a_{k, i} \exp \left(j 2 \pi(k-i) f / f_{s h}\right)
$$

where $a_{k, i}$ denotes the filter coefficient, $i$ is the line for which the flow signal is extracted and $k$ denotes the lines that enter the filtration, and $N_{a v e}$ is the total number of lines. Note that this expression is also valid for non-causal filters in which lines both pre- and proceeding the actual line are used. It

$$
R_{\mathrm{Snr}}=\sqrt{\frac{4 \sqrt{2}+2 \exp \left(-\frac{2}{B_{r}^{2}}\right)}{2 \sqrt{2}+\exp \left(-\frac{2}{B_{r}^{2}}\right)\left[1-\exp \left(-\left(\frac{\pi B_{r} f_{0}}{\sqrt{2} f_{s h}}\right)^{2}\right)\right]-2 \sqrt{2} \exp \left(-\left(\frac{\pi B_{r} f_{0}}{f_{s h}}\right)^{2}\right) \cos \left(2 \pi \frac{f_{0}}{f_{s h}}\right)}}
$$




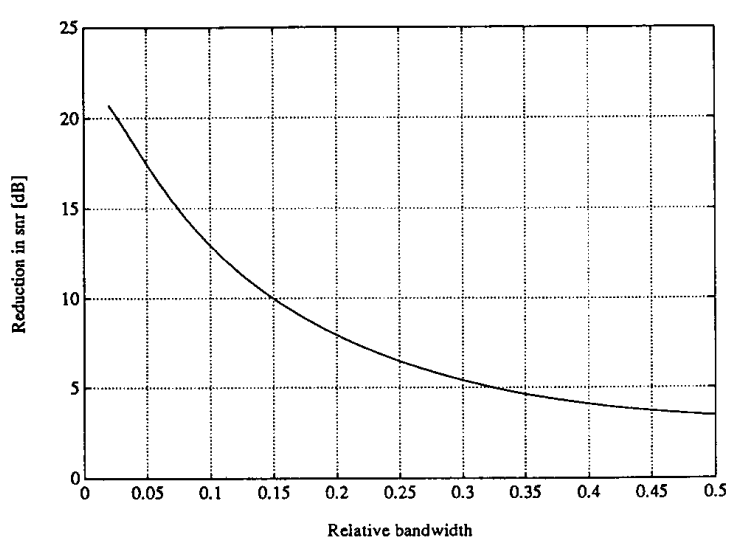

Fig. 6. Reduction of the signal-to-noise ratio as a function of relative transducer bandwidth $B_{r}$.

must, however, be emphasized that the comments put forward about the simple filter still hold. A more advanced filter must also have a zero at $f=0$, and this will introduce zeros in the transfer function at multiples of $f_{s h}$, which influences the signal-to-noise ratio. The influence can, however, be reduced by making the cut-off around $f=0$ sharper.

The filtering of the lines must be done with the same filter for all lines, as the phase change must be the same. Therefore, if a filter with $N_{f}$ coefficients is used and $N_{\text {ave }}$ lines are present, only $N_{\text {ave }}-\left(N_{f}-1\right)$ lines can be used in the subsequent cross-correlation process due to edge effects.

\section{Probability of CoRrect Estimation}

In the time domain cross-correlation method, the velocity is found by first cross-correlating successive A-lines and then finding the position of the maximum peak in the crosscorrelation estimate. The velocity is then related to the time shift $t_{s}$ by [4]:

$$
v=\frac{t_{s}}{T_{p r f}} \frac{c}{2 \cos (\Theta)}
$$

The estimator is nonlinear due to the maximum detection, and it is quite probable that a wrong peak is detected, so erroneous velocities can result from the estimation procedure. In the case of a low signal-to-noise ratio it is appropriate to judge the estimator by the amount of correct detections, or, more formally, the probability of correct detection. We will here define the probability of correct detection as the fraction of estimates that are equal to the true velocity within an interval of $\pm 2.5 \%$ of the maximum detectable velocity. The probability is influenced by the transducer bandwidth, the size of the range gate, or, rather, integration time (number of samples), when calculating the cross-correlation function, the number of A-lines, and signal-to-noise ratio. When stationary echo canceling is used, the velocity will also influence the probability, as the signalto-noise ratio then is velocity-dependent, as shown in the preceding section.

Lacking exact relations linking parameter variations to the probability, a simple simulation program is introduced for
TABLE I

Standard Simulation Parameters

\begin{tabular}{lll}
\hline Parameter & Symbol & Value \\
\hline Transducer center frequency & $f_{0}$ & $3.0 \mathrm{MHz}$ \\
Relative transducer bandwidth & $B_{r}$ & 0.2 \\
Sampling frequency & $f_{s}$ & $20 \mathrm{MHz}$ \\
Propagation velocity & $c$ & $1540 \mathrm{~m} / \mathrm{s}$ \\
Pulse repetition frequency & $f_{p r f}$ & $3200 \mathrm{~Hz}$ \\
Samples in segment & $N$ & 32 \\
Lines for one estimate & $N_{a v e}$ & 4 A-lines \\
Signal-to-noise ratio & $\mathrm{snr}$ & 1 \\
Velocity & $v$ & $1 \mathrm{~m} / \mathrm{s}$ \\
Number of estimates & $N_{\text {trials }}$ & 10000 \\
\hline & &
\end{tabular}

studying the effect of the different parameters on the probability of correct detection. The program simulates movement toward or away from the transducer. The blood scatterers are modeled as a white, zero mean random signal with a Gaussian amplitude distribution. The received signal is the scattering signal convoluted with a one-dimensional transducer pulse with subsequent addition of zero mean, white Gaussian noise. The noise is uncorrelated from line to line. The simulation program performs the following steps:

1. Generate the scattering signal by using a Gaussian random number generator.

2. Time-shift the scattering signal in accordance with the velocity and the pulse repetition frequency.

3. Convolute with the transducers pulse-echo impulse response.

4. Add noise to the signal received.

5. Make stationary echo canceling by subtracting successive A-lines.

Steps 2 to 4 are performed as many times as the number of lines entering a single cross-correlation estimate. Step 5 is performed $N_{\text {ave }}-1$ times, so one line is lost due to the stationary echo canceling.

The program mimics the best possible measurement situation in which the movement is purely in the axial direction, thus neglecting effects from transverse motion such as beam intensity modulation or diffraction. The program, however, simulates the basic mechanisms generating the received signal. Being this simple, it is suited to uncover the statistical properties of the estimator for the ideal measurement situation, so this establishes the upper limit on performance.

The standard simulation parameters are given in Table I, and the pulse is given by (11) properly shifted in time to compensate for its non-causality.

It has been suggested that use of only the sign of the data acquired is sufficient to estimate the cross-correlation function [5]. This is indeed possible at only a slight reduction in performance. This eases the implementation considerably, making it possible to construct the electronics with a few inexpensive components and still attain real-time processing.

Fig. 7 shows the results from running the simulation program. All four cases of using full-precision data or the sign and making echo canceling or not are shown. The top graph shows 
the marked influence from the noise, where signal-to-noise ratios below $6 \mathrm{~dB}$ give rather unreliable estimates, when echo canceling is employed. A somewhat surprising result is that the curves for the full precision data do not approach one, but level off at around 0.9 and 0.7 , respectively. This is due to edge effects. There is a certain probability that strongly reflecting scatterers will enter the volume under investigation during data acquisition, and, thus, dominate the cross-correlation and create a false peak. The effect is most pronounced when echo canceling is done due to the elongation of the pulse from the subtraction of two time-shifted pulses. The effect is not present for the one-bit correlation, as this does not take amplitude into account.

A marked difference is also seen in the probability of correct detection as a function of velocity, when stationary echo canceling is used. From being relatively insensitive to variation in velocity, the probability gets strongly velocitydependent. The variation closely follows the graph given in Fig. 5, and most notable is the near zero probability of estimating velocities around $0 \mathrm{~m} / \mathrm{s}$.

The cross-correlation function estimate can be smoothed by employing a number of lines. This increases the effective integration time and, thereby, reduces the influence from noise, and, thus, increases the probability as shown in the third graph in Fig. 7.

The influence from a variation in transducer bandwidth is shown in the fourth graph of Fig. 7. An increase in bandwidth lowers the side lobes of the autocorrelation of the pulse and increases the probability. The last graph shows that an increase in segment length or integration time increases the probability of correct detection, when assuming a uniform velocity in the segment.

For all curves a lower probability is seen when using only the sign of the data. The reduction is, however, so small that using the sign is a viable alternative making the implementation of the technique considerably easier and cheaper.

\section{A RELIABILITY INDEX}

From the previous discussion it is evident that quite erroneous velocity estimates can arise. The probability of correct detection depends on the measurement situation, most notable on the signal-to-noise ratio and on the actual velocity, both of which are unknown at the time of estimation. It is, therefore, of paramount importance to have an index indicating the reliability of the estimate at hand, and the index should be calculated from the actual data involved in estimating the velocity.

Velocities very different from the true velocity can be estimated because the estimate of the cross-correlation function is perturbed due to noise in the acquired data. This can make the side lobe peaks in the cross-correlation function larger than the peak at the true velocity. The noise introduces a spread of the energy in the cross-correlation function estimate away from the correct peak, and this spread reduces the amplitude of the peak.
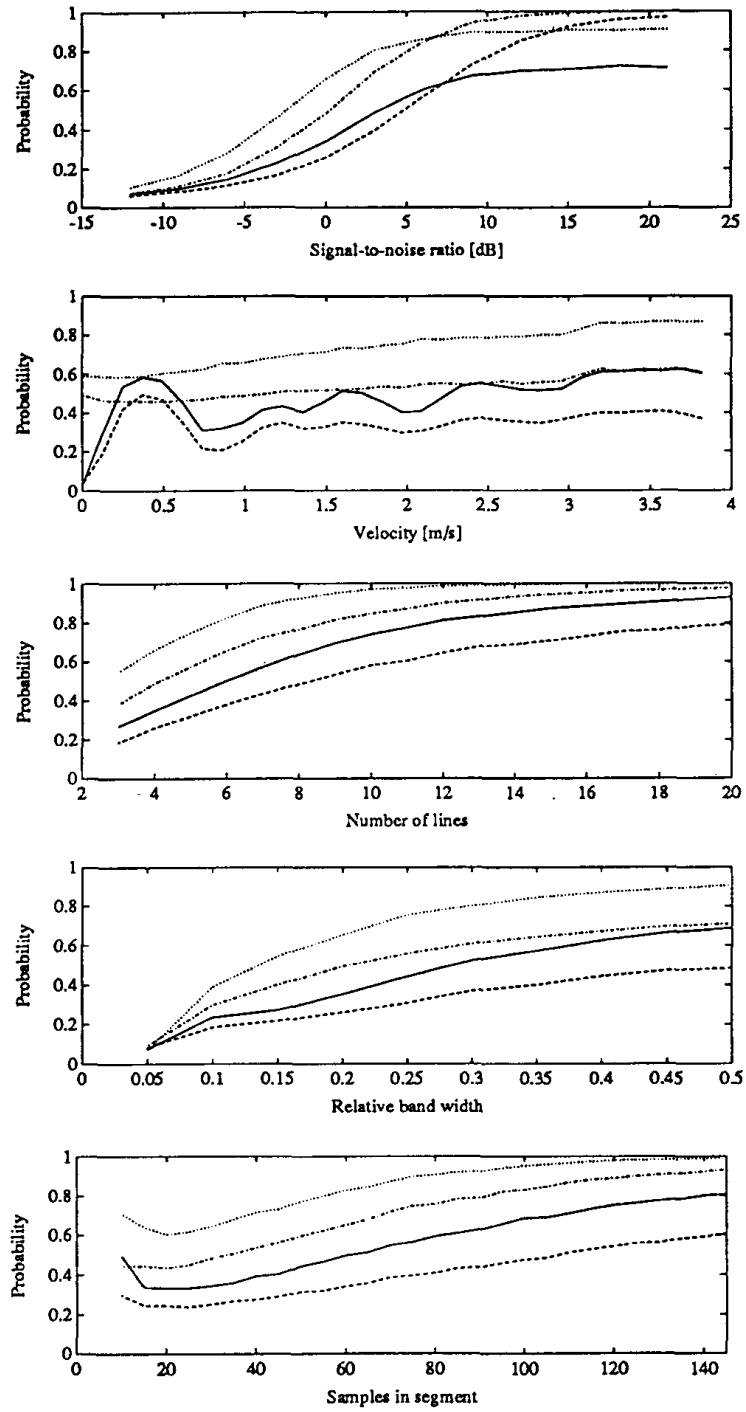

Fig. 7. Variation in probability of correct detection due to different values of the parameters. (-) Full-precision data and echo canceling. (- - ) Sign and echo canceling. $(\cdots)$ Full-precision data without echo canceling. $(\cdots)$ Sign data without echo canceling.

The discrete cross-correlation function estimate is calculated by

$$
\hat{R}_{12}(n)=\frac{1}{N} \sum_{k=0}^{N-1} \operatorname{sgn}\left[y_{1}(k)\right] \operatorname{sgn}\left[y_{2}(k+n)\right]
$$

where

$$
\operatorname{sgn}[y(k)]=\left\{\begin{aligned}
1 & \text { for } y(k) \geq 0 \\
-1 & \text { for } y(k)<0
\end{aligned}\right.
$$

if only the sign of the data is used. $N$ is the number of samples in one segment (range gate). If noise is neglected and $y_{2}$ is an ideal time-shifted replica of $y_{1}$, then the peak value of $\hat{R}_{12}(n)$ becomes 1 , as identical sample values are multiplied. The peak value of $\hat{R}_{12}(n)$ will be less than one if $y_{2}$ is different from 


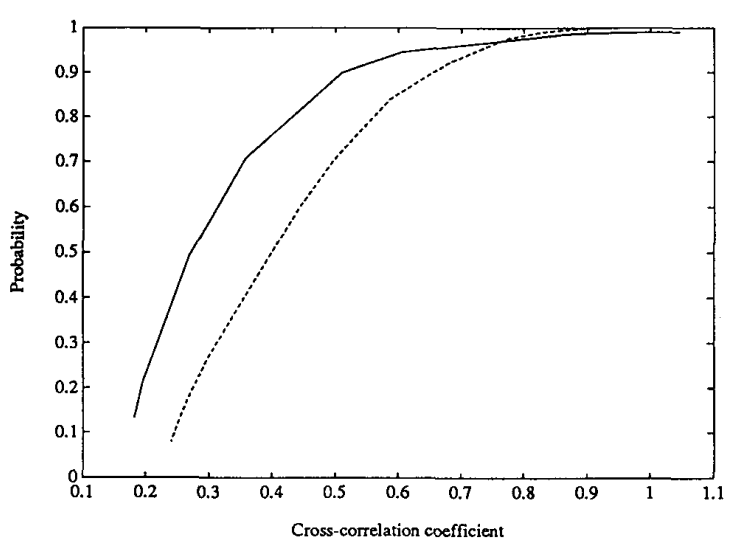

Fig. 8. Relation between reliability index and probability of correct detection $\left(I_{r d}-, I_{\left.r s^{-}--\right)}\right.$.

$y_{1}$ due to noise. With this in mind, a possible index for the reliability could be

$$
I_{r s}=\max \left\{\hat{R}_{12 s}(n)\right\}
$$

where $\max \{\}$ denotes the maximum value of the function. An index close to 0 indicates a very unreliable estimate, and an index close to 1 indicates a very reliable estimate. The index is the cross-correlation coefficient between $y_{1}$ and $y_{2}$, that indicates how similar the two signals are, where one indicates that they are equal.

That the averaged index is highly correlated with the probability of correct detection is shown in Fig. 8 as the dashed line. The data shown is the average for 10000 estimates at each set of parameter values.

When using the full data, the index becomes slightly more complex:

$$
\begin{aligned}
I_{r d} & =\frac{\max \left\{\hat{R}_{12}(n)\right\}}{\sqrt{\hat{P}_{1} \hat{P}_{2}}} \\
\hat{P}_{x} & =\frac{1}{N} \sum_{k=0}^{N-1} y_{x}^{2}(k), \quad x=1,2
\end{aligned}
$$

Here the power of the two signals need also be calculated. This index is shown as the solid line in Fig. 8.

From these indices a direct decision on the reliability or probability of correct detection can be made. A possible method could be to discount estimates with an index below e.g. 0.6 and then use color intensity modulation to show the reliability of the displayed velocity estimates. Another possibility is to adapt the averaging process of the correlation function estimate by employing more or less lines depending on the index to optimize on either time efficiency or reliability for the data at hand.

The curves in Fig. 8 show the averaged indices for 10000 realizations at the given parameters yielding a close correspondence between index and probability. But the individual values of the index will fluctuate around a mean value as shown in Fig. 9. This fluctuation makes the index of little value, when a single estimate of it is used. But at a given range, the signalto-noise ratio will be constant, so averaging can be performed.

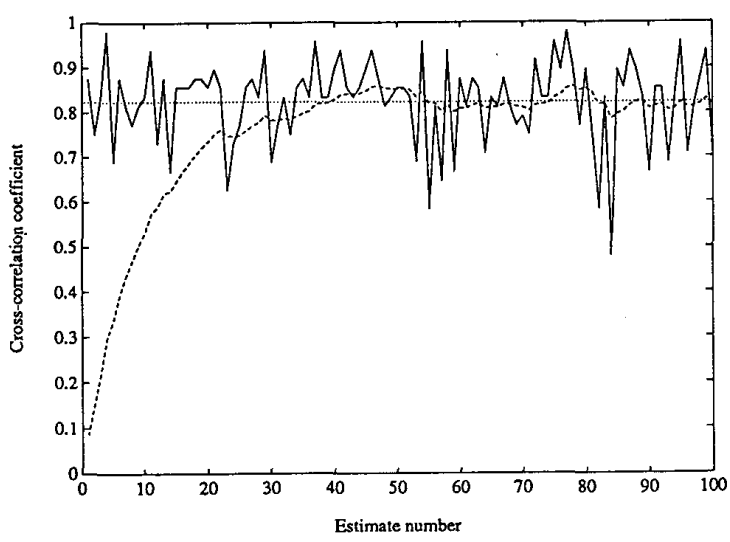

Fig. 9. Reliability index $I_{r s}$ as a function of time ( - individual estimates, -. - averaged index, ... mean value).

A recursive smoothing could be implemented by

$$
\hat{I}_{r m}(i)=\lambda \hat{I}_{r m}(i-1)+(1-\lambda) \hat{I}_{r}(i)
$$

where $\hat{I}_{r}(i)$ is the current estimate of the index and $\hat{I}_{r m}$ is the smoothed index. $\lambda$ is the filter constant determining the time horizon of the averaging. It corresponds to a time constant of

$$
\tau_{a}=\frac{1}{1-\lambda}
$$

so a value of 0.9 gives rise to averaging over roughly 10 to 20 estimates. An example of averaging with $\lambda=0.9$ is shown as the dashed line in Fig. 9. After the initial startup phase, a good estimate is found with an acceptable fluctuation around the mean value indicated by the dotted line. If the frame rate of the scanner is 20 pictures per second, an acceptable estimate of $I_{r}$ is found after just one second.

\section{CONCLUSION}

The influence from stationary echo canceling on the estimation of velocities by time-domain cross-correlation was investigated. The removal of stationary echoes from the received signal introduces a velocity dependent filtration of the flow signal, so the signal-to-noise ratio is reduced with a factor depending on the velocity. This in turn influences the probability of detecting the correct velocity. This probability depends on the transducer bandwidth, integration time used in the calculation of the cross-correlation function, number of A-lines, signal-to-noise ratio, and actual velocity.

As the two last factors can not be known ahead of time, it is of great value to have an index that indicates the reliability of the velocity estimate. It was shown that this can be calculated from the estimate at hand as the normalized maximum of the estimated cross-correlation function. The quantity directly indicates the correlation between the two signals, and its mean value is related to the probability of correct detection.

The index fluctuates around a mean value from estimate to estimate, and smoothing is necessary to make it useful for decision purposes. The smoothed index can be used for deciding whether or not to show an estimate, and for showing how reliable the estimates displayed are. This could be done by controlling the intensity of the colors displayed by the index. 


$$
R_{\mathrm{Snr}}=\sqrt{\frac{4 \sqrt{2}+2 \exp \left(-\frac{2}{B_{r}^{2}}\right)}{2 \sqrt{2}+\exp \left(-\frac{2}{B_{r}^{2}}\right)\left[1-\exp \left(-\left(\frac{\pi B_{r} f_{0}}{\sqrt{2} f_{s h}}\right)^{2}\right)\right]-2 \sqrt{2} \exp \left(-\left(\frac{\pi B_{r} f_{0}}{f_{s h}}\right)^{2}\right) \cos \left(2 \pi \frac{f_{0}}{f_{s h}}\right)}}
$$

It should be emphasized that the index is calculated from the actual cross-correlation function estimate. Thereby all factors such as velocity, signal-to-noise ratio, beam intensity modulation [6], migration of scatterers in and out of the beam, and velocity variation inside the measurement volume [8] are included, and the corresponding reliability indicated by the index.

\section{ACKNOWLEDGMENT}

The author would like to express thanks to Dr. Jens E. Wilhjelm, Associate Professors Dr. Lars-Kai Hansen, Peter Koefoed Møller, and Simon Boel Pedersen for many valuable discussions, and to Dr. Jens E. Wilhjelm and Associate Professor Dr. Lars-Kai Hansen for helping in proofreading the paper.

\section{APPENDIX A: DETERIORATION OF THE SIGNAL-TO-NOISE RATIO}

The signal-to-noise ratio is reduced by the echo canceling filter as shown in Section II. The reduction can be written as

$$
\begin{aligned}
R_{\text {Snr }} & =\frac{\sqrt{\frac{E\left[\{p(t) * s(t)\}^{2}\right]}{E\left[n^{2}(t)\right]}}}{\frac{1}{\sqrt{2}} \sqrt{\frac{E\left[\left\{p(t) * h\left(t ; t_{s}\right) * s(t)\right\}^{2}\right]}{E\left[n^{2}(t)\right]}}} \\
& =\sqrt{2} \sqrt{\frac{E\left[\{p(t) * s(t)\}^{2}\right]}{E\left[\left\{p(t) * h\left(t ; t_{s}\right) * s(t)\right\}^{2}\right]}}
\end{aligned}
$$

where $E$ is the expectation operator. $s(t)$ can be substituted by a dirac impulse, as $s(t)$ is white noise, so (21) can be reduced to

$$
R_{\mathrm{Snr}}=\sqrt{2} \sqrt{\frac{E\left[\{p(t)\}^{2}\right]}{E\left[\left\{p(t) * h\left(t ; t_{s}\right)\right\}^{2}\right]}}
$$

Formally the mean value should be replaced by a measure of the energy of these finite duration signals given as $\int_{-\infty}^{\infty} g^{2}(t) d t$. This can be rewritten as

$$
\int_{-\infty}^{\infty} g^{2}(t) d t=\int_{-\infty}^{\infty}\left|G^{2}(f)\right| d f=\int_{-\infty}^{\infty} G(f) G^{*}(f) d f
$$

using Parseval theorem. $G(f)$ is the Fourier transform of $g(t)$, and $G^{*}(f)$ indicates complex conjugate. Eq. (22) can then be written as

$$
R_{\mathrm{snr}}=\sqrt{2} \sqrt{\frac{\int_{-\infty}^{\infty}|P(f)|^{2} d f}{\int_{-\infty}^{\infty}\left|P(f) H\left(f ; f_{s h}\right)\right|^{2} d f}}
$$

The pulse is given by

$$
p(t)=\exp \left(-2\left(B_{r} f_{0} \pi\right)^{2} t^{2}\right) \cos \left(2 \pi f_{0} t\right)
$$

where $B_{r}$ is the relative bandwidth and $f_{0}$ the center frequency. Its Fourier transform is

$$
\begin{aligned}
P(f) & =\int_{-\infty}^{\infty} p(t) \exp (j 2 \pi f t) d t \\
& =\frac{1}{2 B_{r} f_{0} \sqrt{2 \pi}}\left(\exp \left(-\frac{\left(f_{0}-f\right)^{2}}{2\left(B_{r} f_{0}\right)^{2}}\right)+\exp \left(-\frac{\left(f_{0}+f\right)^{2}}{2\left(B_{r} f_{0}\right)^{2}}\right)\right)
\end{aligned}
$$

The Fourier transform of the filter is given by (7). Then $\int_{-\infty}^{\infty}\left|P(f) H\left(f ; f_{s h}\right)\right|^{2} d f$ can be written

$$
\begin{aligned}
& \int_{-\infty}^{\infty}\left|P(f) H\left(f ; f_{s h}\right)\right|^{2} d f \\
&=\int_{-\infty}^{\infty} P(f) 0.5\left(1-\exp \left(j 2 \pi f / f_{s h}\right)\right) \\
& \cdot P^{*}(f) 0.5\left(1-\exp \left(-j 2 \pi f / f_{s h}\right)\right) d f \\
&= \frac{1}{8 \pi\left(B_{r} f_{0}\right)^{2}} \int_{-\infty}^{\infty}\left[\exp \left(-a\left(f_{0}-f\right)^{2}\right)+\exp \left(-a\left(f_{0}+f\right)^{2}\right)\right]^{2} \\
& \cdot\left(1-\exp \left(j 2 \pi f / f_{s h}\right)\right)\left(1-\exp \left(-j 2 \pi f / f_{s h}\right)\right) d f \\
&= \frac{1}{8 \pi\left(B_{r} f_{0}\right)^{2}} \int_{-\infty}^{\infty}\left[\exp \left(-2 a\left(f_{0}-f\right)^{2}\right)\right. \\
&\left.+\exp \left(-2 a\left(f_{0}+f\right)^{2}\right)+\exp \left(-4 a\left(f_{0}^{2}+f^{2}\right)\right)\right] \\
& \cdot\left[2-\exp \left(j 2 \pi f / f_{s h}\right)-\exp \left(-j 2 \pi f / f_{s h}\right)\right] d f
\end{aligned}
$$

where $a=1 /\left(2\left(B_{r} f_{0}\right)^{2}\right)$.

A functionally similar expression can be found for $\int_{-\infty}^{\infty}$ $|P(f)|^{2} d f$. A lot of tedious calculations then lead to (28) [at top of page] where $f_{s h}=\frac{c}{2 v} f_{p r f}$.

\section{REFERENCES}

[1] C. Kasai, K. Namekawa, A. Koyano, and R. Omoto, "Real-time twodimensional blood flow imaging using an autocorrelation technique," IEEE Trans. Sonics. Ultrason, vol. 32, pp. 458-463, May 1985.

[2] L. Hatle and B. Angelsen, Doppler Ultrasound in Cardiology, Physical Principles and Clinical Applications, 2nd. ed. Philadelphia, PA: Lea \& Febiger, 1985.

[3] D. Dotti, E. Gatti, V. Svelto, A. Uggé, and P. Vidali, "Blood flow measurements by ultrasound correlation techniques," Energia Nucleare, vol. 23, Nov. 1976.

[4] O. Bonnefous and P. Pesqué, "Time domain formulation of pulsedoppler ultrasound and blood velocity estimation by cross correlation," Ultrasonic Imaging, vol. 8, pp. 73-85, 1986.

[5] O. Bonnefous, P. Pesqué, and X. Bernard, "A new velocity estimator for color flow mapping," in Proc. IEEE Ultrason. Symp., pp. 855-860, 1986.

[6] S. G. Foster, P. M. Embree, and W. D. O'Brien, "Flow velocity profile via time-domain correlation: Error analysis and computer simulation," IEEE Trans. Ultrason., Ferroelect., and Freq. Contr., vol. 37, pp. 164-175, May 1990

[7] M. I. Skolnik, Introduction to Radar Systems. New York: McGrawHill, 1980.

[8] $\mathrm{O}$. Bonnefous, "Statistical analysis and the time correlation process applied to velocity measurement," in Proc. IEEE Ultrason. Symp., pp. $887-892,1989$. 